\title{
DEFINITIONS OF A GROUP AND A FIELD BY INDEPENDENT
}

\section{POSTULATES*}

\author{
BY \\ LEONARD EUGENE DICKSON†
}

\section{Introduction.}

1. The simple definition here given for a general abstract group relates as to origin and character to Professor Moore's two definitions, Transactions, vol. 3 (1902), pp. 485-492. A few days before the appearance of the addition, Transactions, vol. 5 (1904), p. 549, to his paper, Professor Moore remarked to me that one of his postulates relating to an inverse was redundant, meaning postulate $\left(3_{r}^{\prime \prime}\right)$ of his second definition. I thought the reference was to his first definition and attempted to reconstruct the proof of a redundancy, there absent. This attempt led me to alter his postulate $\left(4_{l}\right)$ to read $a a_{r}^{\prime}=i_{r}$ instead of $a_{\imath}^{\prime} a=i_{r}$ and to note that postulate $\left(3_{l}\right)$ becomes redundant in the altered set, thus obtaining the present definition. Subsequently I learned that Professor Moore, in his proof of the redundancy of $\left(3_{r}^{\prime \prime}\right)$ in his second definition, had obtained relations $\ddagger$ sufficient to establish the present definition but had not applied them to set up the definition itself.

The present postulates for a general group possess the desirable property that they remain independent within sets of postulates for special classes of groups, the specialization being either in the direction of the number $(n>1)$ of elements or their commutativity ( $\$ 3-5)$.

The definition of a field $(\S 6)$, based on the present definition of a group, has evident advantages over the earlier definitions. $\S$

The postulates for a field remain independent under an assumption that the set is finite, or forms an enumerable infinitude, or a non-enumerable infinitude.

\footnotetext{
* Presented to the Society (Chicago) December 30, 1904, under the title "A definition of a group by independent postulates." Received for publication November 19, 1904. The definition of a field was added January 14, 1905.

† Research Assistant to the Carnegie Institution of Washington.

$\ddagger$ Moore, this number of Transactions, p. 179.

§ Huntington and Dickson, Transactions, vol. 4 (1903), p. 31, p. 13 . For the new definitions by Huntington, with a bibliography, see this number of Transactions, p. 181 .
} 
Definition of a group, $\S \S 2-5$.

2. Given a function $a \circ b$ of two arguments and a set of elements, we will say that the elements form a group with respect to $O$ when the following postulates hold:*

$\left(1_{1}\right)$ For every two $\dagger$ equal or distinct elements $a$ and $b$ of the set, there is a determination of $a \circ b$.

$\left(1_{2}\right)$ For $a$ and $b$ in the set, there is at most one determination of $a \circ b$.

$\left(1_{3}\right)$ If, for $a$ and $b$ in the set, there is at least one determination of $a \circ b$, one determination is an element of the set.

(2) $(a \circ b) \circ c=a \circ(b \circ c)$ whenever $a, b, c$, and all the determinations of $a \circ b, b \circ c,(a \circ b) \circ c$, and $a \circ(b \circ c)$ occur in the set.

(3) There occurs in the set an element $i$ such that, for every element $a$ of the set, $a \circ i$ has the determination $a$.

(4) If such elements $i$ occur, then for a particular $i$, and for every $\ddagger a$ in the set there occurs in the set an element $a^{\prime}$ such that $a \circ a^{\prime}$ has the determination $i$.

Postulates $\left(1_{1}\right),\left(1_{2}\right),\left(1_{3}\right)$ may be combined into the triple statement:

(1) For every two equal or distinct elements $a$ and $b$ of the set $a \circ b$ is uniquely determined as an element of the set.

In view of (3) and (4), to any element $a$ there correspond elements $a^{\prime}$ and $a^{\prime \prime}$ such that $a \circ a^{\prime}=i, a^{\prime} \circ a^{\prime \prime}=i$, where $i$ is a fixed element such that $e \circ i=e$ for every element $e$. Applying also (1) and (2), we have

$$
\begin{aligned}
a & =a \circ i=a \circ\left(a^{\prime} \circ a^{\prime \prime}\right)=\left(a \circ a^{\prime}\right) \circ a^{\prime \prime}=i \circ a^{\prime \prime}, \\
a^{\prime} \circ a & =a^{\prime} \circ\left(i \circ a^{\prime \prime}\right)=\left(a^{\prime} \circ i\right) \circ a^{\prime \prime}=a^{\prime} \circ a^{\prime \prime}=i .
\end{aligned}
$$

Hence $a^{\prime \prime} \circ a=i$. By this theorem, $a^{\prime \prime} \circ a^{\prime}=i$. Hence

$$
i \circ a=(i \circ i) \circ a=\left[i \circ\left(a^{\prime \prime} \circ a^{\prime}\right)\right] \circ a=\left(i \circ a^{\prime \prime}\right) \circ\left(a^{\prime} \circ a\right)=a \circ i=a \text {. }
$$

Since $a \circ i=a=i \circ a$ for every $a, i$ is called an identity element. If also $i_{1}$ is an identity element, then $i_{1}=i_{1} \circ i=i$. Hence there is an unique identity element. Since $a \circ a^{\prime}=i=a^{\prime} \circ a, a^{\prime}$ is called an inverse of $a$. If also $a_{1}^{\prime}$ is an inverse of $a$, then

$$
a_{1}^{\prime}=a_{1}^{\prime} \circ i=a_{1}^{\prime} \circ\left(a \circ a^{\prime}\right)=\left(a_{1}^{\prime} \circ a\right) \circ a^{\prime}=i \circ a^{\prime}=a^{\prime} .
$$

Hence there is an unique inverse of each element.

* In (2), (6), and (7), we mean by $A=B$ that one of the determinations of $A$ equals one of the determinations of $B$.

† The assumption need not be made for $a \circ i$ or $a \circ a^{\prime}$, in view of (3), (4).

$\ddagger$ The assumption need not be made for $a=i$, in view of ( 3 ). 
3. We prove that the postulates $\left(1_{1}\right),\left(1_{2}\right),\left(1_{3}\right),(2),(3),(4),\left(5_{k}\right)$, $k=1,2$, or 3 , are consistent and independent, where

$\left(5_{1}\right)$ The number of distinct elements is a fixed integer $n, n>1$; *

$\left(5_{2}\right)$ The distinct elements of the set form an enumerable infinitude;

$\left(5_{3}\right)$ The distinct elements form a non-enumerable infinitude.

Their consistency follows from the existence of the group of the elements $0,1, \cdots, n-1$ with $a \circ b=a+b(\bmod n)$, and the group of all rational (or real) numbers with $a \circ b=a+b$.

Let $I_{1}$ be a set containing $i$ and exactly $n-1$ further elements $b ; I_{2}$ or $I_{3}$ a set containing $i$ and further elements $b$ forming an enumerable or a non-enumerable infinitude, respectively.

To prove the independence of a postulate $(j)$, we exhibit a set $[j]$ in which postulate $(j)$ fails while each of the remaining postulates hold. In the sets, $a \circ b$ is understood to have a unique determination unless the contrary is stated.

$\left[1_{1}\right]$ Set $I_{k}, i \circ i=i, b \circ b=i, b \circ i=b$, no determination of $i \circ b$ or of $b \circ b^{\prime}\left(b \neq b^{\prime}\right)$. and $i$.

$\left[1_{2}\right]$ Set $I_{k}$ forming a group under $\odot ; a \circ c$ with the determinations $a \odot c$

[1] Set $I_{k} ; i \circ i=i, b \circ i=b, b \circ b^{i}=i, i \circ b$ not in the set.

[2] Set $I_{k}$; $i \circ i=i, b \circ i=b, b \circ b^{\prime}=i, i \circ b=i$.

[3] Set $I_{k}$; $i \circ i=i, b \circ i=i, b \circ b^{\prime}=i, i \circ b=i$.

[4] Set $I_{k}$; $i \circ i=i, b \circ i=b, i \circ b=b, b \circ b^{\prime}=b_{1}, b_{1}$ a fixed $b$.

4. We next examine the effect of adding the commutative law :

(6) $a \circ b=b \circ a$ whenever $a, b$, and all the determinations of $a \circ b$ and $b \circ a$ occur in the set.

The postulates $\left(1_{1}\right),\left(1_{2}\right),\left(1_{3}\right),(2),(3),(4),\left(5_{k}\right),(6)$, for $k=0,2$, or 3 , are consistent and independent. Here the new postulate is

$\left(5_{0}\right)$ The number of distinct elements is finite but undetermined.

The proof follows from the sets $\left[1_{1}\right],\left[1_{2}\right],\left[1_{3}\right],[3],[4]$ above, and

$[2]^{\prime}$ Set $I_{k}, n>2 ; i \circ i=i, i \circ b=b \circ i=b, b \circ b=i, b \circ b^{\prime}=b_{1}\left(b \neq b^{\prime}\right)$, Then, for $b \neq b_{1},\left(b \circ b_{1}\right) \circ b_{1}=b_{1} \circ b_{1}=i, b \circ\left(b_{1} \circ b_{1}\right)=b \circ i=b$.

[6] There exist finite and infinite non-commutative groups. $\dagger$

The question of the independence of the postulates for $k=1$ is answered by $\S 5$ in connection with the sets $\left.\left[1_{1}\right],\left[1_{2}\right],\left[1_{3}\right],[2]\right]^{\prime},[3],[4]$.

5. Theorem. $\ddagger$ Let $n=p_{1}^{a_{1}} p_{2}^{a_{2}} \cdots p_{v}^{a_{v}}$, where $p_{1}, \cdots, p_{v}$ are distinct primes, and each $\alpha_{j}>0$. The necessary and sufficient conditions that all existing

* For $n=1$, the group may be definer by the independent postulates $\left(1_{1}\right),\left(1_{2}\right),\left(1_{3}\right)$; also by the independent postulates $\left(1_{2}\right),(3)$.

$f$ E. g., that of the transformations $x^{\prime}= \pm x+b$, where $b$ ranges over all integers, or all real numbers, or the resid nes modulo $m, m>2$.

$\ddagger$ Addition of February 2, 1905. 
groups of order $n$ shall be abelian are: (i) each $x_{j} \equiv 2$; (ii) no $p_{j}^{a_{j}}-1$ is divisible by one of the primes $p_{1}, \cdots, p_{.}$.

We first prove that the conditions are necessary. If $\alpha_{1}>2$, a non-abelian $G_{n}$ is given by the direct product of the cyclic groups $C_{p_{k}}^{a_{k}}(k=2, \ldots, v)$ and a non-abelian $G_{p_{1}^{a_{1}}}$, say of the type generated by $P$ and $Q$ where

$$
P^{p_{1}^{a_{1}-1}}=I, \quad Q^{p_{1}}=I, \quad Q^{-1} P Q=P^{1+p_{1} a_{1}-2} \quad\left(a_{1}>\approx\right) .
$$

If each $\alpha_{j} \leqq 2$ and $p_{1}^{a_{1}}-1$ is dịvisible by $p_{2}$, there exists a non-abelian group of order $p_{1}^{a_{1}} p_{2}^{a_{3}}$ and hence, as before, one of order $n$. Indeed, if $\alpha_{1}=\alpha_{2}=1$, we use the group generated by $P_{1}$ and $P_{2}$ where

$$
P_{1}^{p_{1}}=I, \quad P_{2}^{p_{2}}=I, \quad P_{2}^{-1} P_{1} P_{2}=P_{1}^{\pi} \quad\left[p_{1}=1\left(\bmod p_{2}\right)\right],
$$

where $\pi$ is an existing primitive root of $x^{p_{2}} \equiv 1\left(\bmod p_{1}\right) . \quad$ If $\alpha_{1}=1, \alpha_{2}=2$, we use the direct product of the preceding group by a cyclic $C_{p_{2}}$. If $\alpha_{1}=2$, $\alpha_{2}=1$, the case in which $p_{1}-1$ is divisible by $p_{2}$ is disposed of as before, while for $p_{1}+1$ divisible by $p_{2}, p_{2}>2$, we use the group* generated by $S, T_{1}, T_{2}$, with

$$
T_{1}^{p_{1}}=T_{2}^{p_{1}}=S^{p_{2}}=I, \quad T_{1} T_{2}=T_{2} T_{1}, \quad S^{-1} T_{1} S=T_{2}, \quad S^{-1} T_{2} S=T_{1}^{-1} T_{2}^{b},
$$

where $b$ is an (existing) integer such that $x^{2}+b x+1 \equiv 0\left(\bmod p_{1}\right)$ is irreducible, viz., $b=i+i^{p_{1}}, i$ a mark of the $G F\left[p_{1}^{2}\right]$ belonging to the exponent $p_{2}$. Finally, if $\alpha_{1}=\alpha_{2}=2$, we use the direct product of one of the preceding groups by $C_{p_{2}}$.

It remains to prove that the conditions $(i)$ and $(i i)$ are sufficient to make every $G_{n}$ abelian. To proceed by induction, we assume that this statement is true for every $n^{\prime}=p_{1}^{\beta_{1}} \cdots p_{v}^{\beta_{v}}, \beta_{1} \leqq \alpha_{1}, \cdots, \beta_{v} \leqq \alpha_{v}, n^{\prime}<n$. Hence every subgroup of $G_{n}$ is abelian, so that $\dagger$ either $G_{n}$ is abelian or else $n$ is divisible by at most two distinct primes. But for $n=p, n=p^{2}$; or $n=p_{1}^{\alpha_{1}} p_{2}^{\alpha_{2}}, 0<\alpha_{1} \leqq 2,0<\alpha_{2} \leqq 2$, $p_{1}^{a_{1}} \neq 1\left(\bmod p_{2}\right), p_{2}^{a_{2}} \neq 1\left(\bmod p_{1}\right), G_{n}$ is immediately seen to be abelian. Hence the induction is complete.

\section{Definition of a field, §§ 6-9.}

6. We employ a set of elements and two functions $a \oplus b$ and $a \otimes b$. For $0=\oplus$, postulate $(j)$ of $\S \S 2,4$, with $j=1,2,3,4$, or 6 , is designated $j^{+}$. An element $i$ satisfying $3^{+}$is designated $i_{+}$. For $O=\otimes,(j)$ is designated $j^{\times}$; but $4^{\times}$is assumed only for elements $a$ distinct from each $i_{+}$. An element $i$ satisfying $3^{\times}$is designated $i_{\times}$. As the distributive law we take $\ddagger$

\footnotetext{
* Hölder, Mathematische Annalen, vol. 43 (1893), pp. 345-357. We may use the form by Cole and Glover, A merioa n Ju rnal of Mathematios, vol. 15 (1893), pp. 207-8.

† Miller and Moreno, Transactions, vol. 4 (1903), p. 398.

If we alter $(7)$ to read $a \otimes(b \oplus c)=(a \otimes c) \oplus(a \otimes b)$, we deduce $6^{+}$by taking $a=i_{x}$ and applying $6^{\times}$. Then also $(7)$ follows.
} 
(7) $a \otimes(b \oplus c)=(a \otimes b) \oplus(a \otimes c)$ whenever $a, b, c$, and all the determinations of the functions involved occur in the set.

In view of the theorems in $\S \S 7,8$, we make the definition:* A set of elements forms a field with respect to $\oplus$ and $\otimes$ if postulates $1^{+}, 2^{+}, 3^{+}, 4^{+}, 1^{\times}$, $2^{\times}, 3^{\times}, 4^{\times}, 6^{\times}$, and 7 hold.

7. Theorem. $\dagger$ From $1^{+}, 2^{+}, 3^{+}, 4^{+}, 1^{\times}, 3^{\times}, 6^{\times}, 7$, follows $6^{+}$.

We substitute $\beta \oplus \gamma$ for $a$ in (7), apply $1^{+}, 1^{\times}, 6^{\times}, 7$ and get

$$
(\beta \oplus \gamma) \otimes(b \oplus c)=[(b \otimes \beta) \oplus(b \otimes \gamma)] \oplus[(c \otimes \beta) \oplus(c \otimes \gamma)],
$$

for every $\beta, \gamma, b, c$ in the set. Interchanging $b$ with $\beta, c$ with $\gamma$, and noting that the first member is unaltered in view of $6^{\times}$, we get by $6^{\times}$and $2^{+}$,

$(b \otimes \beta) \oplus(c \otimes \beta) \oplus(b \otimes \gamma) \oplus(c \otimes \gamma)=(b \otimes \beta) \oplus(b \otimes \gamma) \oplus(c \otimes \beta) \oplus(c \otimes \gamma)$,

Since $1^{+}, 2^{+}, 3^{+}, 4^{+}$imply an inverse under $\oplus$, we get

$$
(c \otimes \beta) \oplus(b \otimes \gamma)=(b \otimes \gamma) \oplus(c \otimes \beta) .
$$

Taking $\beta=\gamma=i_{\times}$, we get $c \oplus b=b \oplus c$.

8. Theorem. Let $i_{+}$be chosen so that $4^{+}$, as well as $3^{+}$, is satisfied. Then $a \otimes i_{+}=i_{+} \otimes a=i_{+}$for every element $a$. If $a \otimes b=i_{+}$and $b \neq i_{+}$, then $a=i_{+}$. Hence $i_{+}$has the ordinary propertiee of zero under $\oplus$ and $\otimes$.

By 7 for $b=c=i_{+}, e=e \oplus e$, where $e=a \otimes i_{+}$. Hence ( $\left.\$ 2\right), a \otimes i_{+}=i_{+}$ for every $a$. By $1^{\times}, 6^{\times}, i_{+} \otimes a=i_{+}$.

By $2^{\times}$, from $a \otimes b=i_{+}$follows $i_{+}=a \otimes(b \otimes c)$ for every $c$. Let $i_{\times}$be chosen so that $4^{\times}$, as well as $3^{\times}$, holds. Taking $c$ such that $b \otimes c=i_{\times}$, we get $i_{+}=a \otimes i_{\times}=a$.

9. Theorem. Postulates $1^{+}, 2^{+}, 3^{+}, 4^{+}, 1^{\times}, 2^{\times}, 3^{\times}, 4^{\times}, 6^{\times}, 7,5_{k}(k=0$, 2 , or 3 ) are independent.

For $k=0$, i. e., for a finite number of elements, we employ the sets :

$\left[1^{+}\right]$Elements $0,1,-1 ; a \oplus b=a+b, a \otimes b=a \times b$.

$\left[2^{+}\right] 0,1,-1 ; 0 \oplus a=a \oplus 0=a, a \oplus b=0(a \neq 0, b \neq 0), \otimes=\times$.

$\left[3^{+}\right] 0,1, \cdots, n-1(n>1) ; a \oplus b=b, a \otimes b=a+b(\bmod n)$.

* If the set is finite, we may omit $3^{+}$and insert $6^{+}$. Then if $3^{+}$fails, all the elements form a group with respect to $\otimes$. It cannot coutain an element $a$ of period $\alpha, \alpha>1$. Indeed, if we set $y=i_{\times} \oplus a \oplus a^{2} \oplus \cdots \oplus a^{a-1}$, we get $a \otimes y=y$, whence $a=i_{\times}$. The single element in the sec necessarily satisfies $3^{+}$.

† Some nonths alter devising this prool I learned that a similar proof had been given hy Hilbert, Jahresbericht der Deutsohen Matbematiker-Vereinigung, vol. 8 (1899$1000)$, p. 183 . But earlier writers have noted the essential point in the proof ; viz., that the uniqueness of the expansion of $(\beta+\gamma)(b+c)$ dependis upon the validity of the commutative law for addition. 
$\left[4^{+}\right]$

\begin{tabular}{|c|c|c|c|}
\hline$\oplus$ & $\begin{array}{ll}0 & 1\end{array}$ & $\otimes$ & 0 \\
\hline 0 & 0 & 0 & 0 \\
\hline 1 & 11 & 1 & 0 \\
\hline
\end{tabular}

$\left[1^{\times}\right] 0,1 ; a \oplus b=a+b(\bmod 2), 0 \otimes a=0,1 \otimes 1=1,1 \otimes 0$ not in the set.

$\left[2^{+}\right]$Eight * elements $(\xi, \eta, \zeta), \xi, \eta, \zeta$ taken modulo 2 ;

$$
(\xi, \eta, \zeta) \oplus(x, y, z)=(\xi+x, \eta+y, \zeta+z),
$$

$(\xi, \eta, \zeta) \otimes(x, y, z)=(z \xi+x \zeta,(x+y) \xi+(x+z) \eta+y \zeta, y \xi+x \eta+z \zeta)$. The latter equals $i_{\times}=(0,0,1)$ if $D \equiv \xi+\zeta+\xi \zeta \neq 0(\bmod 2)$ and

$$
x \equiv \xi(1+\zeta) / D, y \equiv(\xi+\xi \eta+\eta \zeta) / D, z \equiv \zeta(1+\xi) / D .
$$

But $D \equiv 0$ only when $\xi \equiv \zeta \equiv 0$; while if $\xi \equiv \zeta \equiv 0, \eta \equiv 1$, we have

$$
(0,1,0) \otimes(x, y, z)=(0, x+z, x)=i_{\times} \text {if } x \equiv z \equiv 1 \text {. }
$$

Finally, $2^{\times}$fails for $a=(0,1,0), b=(0,1,0), c=(1,1,1)$.

$\left[3^{\times}\right] 0,1 ; a \oplus b=a+b(\bmod 2), a \otimes b=0$.

$\left[4^{\times}\right] 0,1, \cdots, n-1, n$ composite; $a \oplus b=a+b(\bmod n), a \otimes b=a \times b$ $(\bmod n)$

$\left[6^{\times}\right]$Nine elements $a+b j, a, b \equiv 0,1,-1(\bmod 3)$;

$$
\begin{gathered}
(a+b j) \oplus(c+d j)=a+c+(b+d) j, \\
(a+b j) \otimes(c+d j)=a c-\cdot(-1)^{a b} b d+\left\{b c+(-1)^{a b} a d\right\} j,
\end{gathered}
$$

where the exponent $a b$ is taken in the form 0,1 , or -1 . Then $j \otimes j=-1$,

$$
j \otimes(1+j)=-1+j, \quad(1+j) \otimes j=1-j,
$$

so that $6^{\times}$and the right-hand distributive law fail. For $4^{\times}$,

$$
(a+b j) \otimes\left(\frac{a-(-1)^{a b} b j}{a^{2}+b^{2}}\right)=1=i_{\times},
$$

since $a^{2}+b \equiv 0$ only when $a \equiv b \equiv 0(\bmod 3)$. Computation shows that $2^{\times}$holds.

[7] Any finite group of order $>1 ; a \oplus b=a \otimes b=a \circ b$.

[5] All rational numbers; $\oplus=+, \otimes=x$.

* A much simpler statement of $\Sigma_{7}$, Transactions, rol. 4 (1903), p. 20 . 
For $k=2(k=3), i$. e., for an enumerable (a non-enumerable) infinitude of elements, we employ the following sets $[j], R$ denoting the set of all rational (real) numbers, $R_{+}$that of all positive rational (positive real) numbers :

[1+] $R ; \otimes=\times, a \oplus 0=0 \oplus a=a, a \oplus a=0, a \oplus b$ not in $R$ if $a \neq b$, $a \neq 0$ or $b \neq 0$.

$\left[2^{+}\right] R ; \otimes=\times, a \oplus a=0, a \oplus b=a+b(a \neq b)$.

$\left[3^{+}\right] R_{+} ; \oplus=+, \otimes=\times$.

[4+] $R_{+}$and zero; $\oplus=+, \otimes=\times$.

[ $\left.1^{\times}\right] R ; \oplus \equiv+, a \otimes b=a b($ if $a=1, b=1$, or $a b=1$ ), otherwise $a \otimes b$ not in $R$.

$\left[2^{\times}\right]$Hypercomplex numbers $\alpha+\beta i+\chi j, \alpha, \beta, \gamma$ arbitrary in $R$;

\begin{tabular}{r|rrr}
$\otimes$ & 1 & $i$ & $j$ \\
\hline 1 & 1 & $i$ & $j$ \\
$i$ & $i$ & -1 & 1 \\
$j$ & $j$ & 1 & -2
\end{tabular}

Then $(i i) j=-j, i(i j)=i ;(\alpha+\beta i+\chi j)(x+y i+z j)=1$ if $x=\alpha / \Delta, \quad y=-\beta / \Delta, \quad z=-\gamma / \Delta, \quad \Delta=\alpha^{2}+\gamma^{2}+(\beta-\gamma)^{2}$.

[3×] $R ; a \oplus b=a+b, a \otimes b=0$.

$\left[4^{\times}\right]$Positive and negative integers and zero; $\oplus=+, \otimes=x$. $a_{1} e_{1}+a_{2} e_{2}$ with $a_{1}$ and $a_{2}$ real, $e_{1}^{2}=0, e_{1} e_{2}=e_{2} e_{1}=e_{1}, e_{2}^{2}=e_{2} ; \oplus=+$, $\otimes=\times$; then $e_{1} \otimes y=i_{\times}=e_{2}$ is impossible since $\left.e_{1}\left(y_{1} e_{1}+y_{2} e_{2}\right)=y_{2} e_{1} \cdot\right)$

[6] All quaternions $a i+b j+c k+d, a, b, c, d$ in $R ; \oplus=+, \otimes=\times$

[7] $R_{+} ; \oplus=\otimes=\times$.

$\left[5_{k}, k=2\right.$ or 3$]$. Any finite field, e. $g$., the classes of residues modulo $p$.

The University of Chicago. 